\title{
Handover Management Optimization over LTE -A Network using S1 and X2 handover
}

\author{
H.A. Sakr, M.A. Mohamed
}

\begin{abstract}
Handover is one of the essential components in the mobility management of wireless systems. It allows the client equipment (UE) to connect between LTE-A networks. The variables that can influence this handover performance are the kind of interfaces in which the handover exists, either $\mathrm{X} 2$ or S1 interface, the mobility of the UE and the QCI (Quality of Service Class Identifiers). S1 interface associates the eNodeB to the EPC. It divided into double interfaces, one for the user plane and the other for the control plane. The mobility within LTE-A/SAE as well as mobility to other systems using both 3GPP and non-3GPP technologies. X2 is a new kind of interfaces introduced by the LTE-A Radio Access Network. Neighboring eNodeBs are linked in a peerto-peer fashion to perform handover and provide a means for the rapid coordination of radio resources. This research looks at the handover parameters (e.g. EPS bearer bit loss, BLER, throughput, SNR, LTE-A delay) on the mobile user and eNodeBs. Performance of three different applications measured, namely, Video data streaming, Voice traffic and HTTP web browser running on a mobile user were evaluated with a background traffic. This case study proposes the two types of handover $\mathrm{X} 2$ and $\mathrm{S} 1$ parameters to achieve a maximum possible quality of multimedia streaming services. Furthermore, discuss the effectiveness of using X2 handover and S1 handover on the overall network performance. Using OPNET Simulator 17.5, it concluded that X2 handover is preferred to operate compared with the $S 1$ handover, also is a very good choice for all multimedia services and the overall network performance.
\end{abstract}

Keywords- LTE/SAE; Security; Key Management; X2 Handover; Mobility Management LTE-Advanced; handover decision; interference; and OPNET Modeler 17.5.

\section{Introduction}

Mobile information continuous development rises effective advancements to fulfill the required quality of service (QoS) of the new services. Mobility is one of the features of the next generation of cellular systems that enables users to change seamlessly their point of connections while using their data and voice services. Handover in Long Term Evolution Advanced (LTE-A), as in the previous generation of cellular systems, is a strategy to exchange a client equipment (UE) and its setting from a source evolved (eNB) to a target eNB.

M.A. Mohamed

Electronics and Communications Engineering Department- Faculty of Engineering-Mansoura University, Mansoura-Egypt

H.A. Sakr

ECE-Department- Institute of Public Administration- Abha- Saudi Arabia
It requires efficient handover choice algorithms in order to optimize performance for the network and UE and quality. although the non-radio component also evolved which is PAGE 6 now known as the system architecture evolution (SAE) [1].

In 2014, Qing showed a mobility scheme proposed using the optical packet switching. explained in this paper the Optical Packet-based Mobility Management (OPMM) is demonstrated more proficient for mobile movement transport contrasted with traditional GPRS Tunneling Protocol (GTP) in regards to packet overhead likewise transport delay and power consumption. Then, demonstrated that OPMM decreases the unpredictability of the mobility management methodology. The framework configuration is approved in a simulator and the performance is contrasted with the standard 3GPP usage in real scenarios with a reference design [2].

In 2015, Donghyuk et al. [3] exhibited the theoretical performance and a few actual measurements on reasonable LTE Handover. In this paper, the handover latency and handover interrupt time (HIT) execution of LTE systems are estimated for a few cases as per the normal number of clients in a cell, likewise Based on the interior handover strategies that impact handover delay and HIT. What's more, in light of the evaluated number of clients in a cell, a reference probability density function (PDF) that can be utilized for HIT expectation is exhibited. The analysis demonstrates that RACH stack is a key parameter that service providers need to consider since the HIT will increment when the RACH stack increments. To enable adaptive control, the impacts on HIT are estimated and stochastically demonstrated as far as RACH stack, which is spoken to by the quantity of UEs per cell.

In 2016, Konstantinos et al. [4], talked about the Handover methodology in LTE/LTE-A which have been drastically developed contrasted with the past 3GPP models. X2 handover likewise presented to enable neighboring eNBs to deal with the client mobility without the association of the core network. Authors also analyzed the execution of the $\mathrm{X} 2$ handover from the UE point of view. Results demonstrated that the uplink synchronization method got through the Medium Access Control (MAC) RACH technique contributes most to the total latency, and that the contention-free preambles are preferred to avoid the collision, especially in high load and mobility scenarios, particularly in high load and mobility situations.

In 2017, Bangyi et al. [5], introduced the commercialization of LTE/SAE advances begun a new era in which information can be transmitted at amazingly high 
rates. What's more, examined the security of the LTE/SAE network. This research was directed to present forward key security in X2 handover key management in scenarios in which an adversary controls a legal base station. By changing parameters in the renewing step and adding a verification step. Likewise analyzed the security and performance of our proposition with other comparative plans. The upgrade scheme guarantees forward partition security accompanied by favorable signal and computation load performance.

This paper will cover S1 and X2 handover in LTE-A which occurs between eNBs. In the majority of the cases, both source and target eNBs are associated with the same MME and are situated in a similar tracking area (TA). The measurement cases cover a comparative study between the S1 handover and X2 handover. The goal of the paper is to analyze, characterize, and differentiate between the performance of the X2 handover and S1 handover network. The contribution of this study can be listed as follows: (I) Throughput: during multimedia transmission, high amount of data will be dropped so the quality of data received will be very bad, by using X2 handover-based LTE-A, it can satisfy higher throughput rates. (II) Delay reduction: all multimedia applications suffer from higher delay ranges during data transmission so over LTE-A and by using X2 handover we will reduce delay rates under conditions of increasing coverage and mobility. (III) Increasing coverage area: using X2 handover to access multimedia anywhere and by increasing the distance, this will reduce delay problem and with excellent performance and (IV) Bandwidth: By using X2 handover, we will overcome the bandwidth limitations faced by multimedia during transmission. Additionally, this paper represents the strength points by using X2 handover in comparison with the S1 handover. This enables us to improve the multimedia QoS parameters also increasing the speed of multimedia access with higher rates of SNR and lower rates of BER. These results lead to reduce the packet loss during transmission and improve the overall network performance using X2 handover. The other sections of this paper are organized as follows: Section 2 introduces the LTE-A handover types and procedures. Section 3 presents the system QoS Performance Metrics. Section 4 includes the system evaluation., Section V presents the general discussion of simulation results. Finally, Section 5 provides concluding remarks and future directions.

\section{X2 and S1 Handover}

Handover architecture, deployment, and implementation have entirely changed compared to the legacy 3GPP technologies. In LTE-A Evolved Packet System (EPS), RNC has been removed and the intelligence is kept in the eNB side that is responsible for handover .X2 interface can be established between one eNB and its neighbors in order to exchange the intended information. Hence, fully mesh topology is not mandated to the S1 interface where a star topology is used; Moreover, the protocol structure over X2 interface includes each the control and the data plane protocol stack that is similar to over the $\mathrm{S} 1$ interface as depicted in figure 1 and figure 2, The X2 topology in addition to the X2-AP structure provide benefits associated with the information forwarding operation. In case of X2 interface isn't configured or the connection is blocked; handover can be accomplished through MME using $\mathrm{S} 1$ interface. The mobility procedures over the radio interface. These mobility procedures also involve the network interfaces [6].

Mobility over X2 and S1 can be differentiated in four extraordinary modes consistent with the RAB quality class Indicator (QCI). The source eNB has to select based on the UE QoS necessities received (e.g., guaranteed Bit rate (GBR)/non-GBR traffic etc.). These modes are described as follows : (I) control plane: most effective stream control Transmission Protocol (SCTP) connection is established some of the two eNBs for control plane messaging and no information forwarding through X2 interface is supported. in that case, all of the packets that are supposed to be transmitted via the S1 path buffered domestically, but not yet stated by way of the UE). (II) DL data plane: General Packet Radio Service (GPRS) Tunneling Protocol (GTP) tunnels can be mounted for downlink information forwarding on in keeping with radio access bearer. The $\mathrm{X} 2$ request message that is sent via the source eNB proposes the GTP tunnel established order; then the tunnel endpoint is blanketed within the $\mathrm{X} 2$ request ACK message if the established order is wellknown via the target eNB.As a consequence, the source eNB can begin the packet forwarding procedure in parallel with the HO command transmission to the UE. This kind of information forwarding consists of packets arriving over the source S1 direction and is called "seamless handover". As an enhancement, packets which are PDCP processed also can be forwarded (PDCP SN is included in the GTP extension header). The aforementioned data forwarding is referred to as "lossless handover", for the reason that there's no packet loss. (III)UL data plane: Uplink forwarding may be similarly handled by using taking into consideration the traffic coming from the UE aspect that is percent buffered, non-acknowledged by the source eNB and consequently non-forwarded via the $\mathrm{S} 1$ route. This mode is called "selective retransmission", for the reason that UE can be informed by using the target eNB for not re-transmitting those packets accelerating the uplink re-transmission. And (IV) DL \& UL data plane: A combination of the above modes may be additionally executed reducing the general delay. Observed with the control plane messaging assures the general packet transmission both for DL/UL maintaining the handover process seamless to the UE aspect [7].

\section{QoS Performance Metrics}

It's essential to study more powerful standardized metrics that affects directly on the overall system performance as delay and network's throughput as they considered some critical parameters for data transmission system.

\section{A. End to End Delay}

Packet delay, DE2E, is calculated using the following equation [8]:

$D_{\text {E2E }}=Q\left(d_{\text {proc }}+d_{\text {queue }}+d_{\text {trans }}+d_{\text {propa }}\right)$

where $\mathrm{Q}$ is the number of network components between the portable station and application server, dproc is the processing delay at given network, dqueue is presented the queuing delay at any given network component, dtrans is meant the transmission time of a bundle on a given connection between two network components and dpropa is the propagation delay over a given network 
interface. Delay or latency could be defined as the time taken by the packets to reach from source to destination. The small amount of delay does not directly affect the QoS of the Video Streaming. While the delay large than $300 \mathrm{~ms}$ may result from a much worse QoS toward enduser experience. The delay for one way must be less than $300 \mathrm{~ms}$. On the other hand, the end-to-end delay more than $500 \mathrm{~ms}$ is considered unacceptable.

\section{B. Packet Delay Variation}

Packet delay variation (PDV) (measured in $\mathrm{msec}$ ) is defined as the variability in packet delay within a given media at video client station. Eq.2 describes PDV for video streaming process with acceptable ranges $<50$-msec [11].

$$
\mathrm{j}_{\text {pkt }}=\mathrm{t}_{\text {actual }}-\mathrm{t}_{\text {expected }}
$$

where $\mathbf{t}_{\text {actual }}$ is the actual packet reception time; and $\mathbf{t}_{\text {expected }}$ is the expected packet reception time [9].

\section{Average Throughput}

Normal throughput (estimated in packets/sec) is the measure of the number of packets effectively delivered in a network. The value of throughput should be high otherwise it will affect every service class defined in Normal throughput (estimated in packets/sec) is the measure of a number of packets effectively conveyed in a network. The average throughput is defined as follow:

Throughput $=\frac{\sum_{1} \text { packet delivered }}{\sum_{1} \text { packet delivered }- \text { packet start time }}$

Is the rate of the fruitful message transmitted over a correspondence channel. The information these messages have a place with might be delivered over a physical or logical connection or it can go through a specific network node. Throughput is generally estimated in bits every second (packets or bps), and here and there in information packets every second $(\mathrm{p} / \mathrm{s})$ or information packets per availability [9].

\section{Packet Loss Ratio}

Packet Loss Ratio (PLR) is the undermined/lost or too much-delayed packets partitioned by the aggregate number of packets expected at the video customer station. The PLR for the total packet sent and received as follows [10]:

PLR $=\frac{\text { Lost }_{\text {packets }}}{\text { Lost }_{\text {packets }}-\text { Recieved }_{\text {Packets }}}$

\section{E. Mean Opinion Score (MOS)}

MOS provides a numerical measure of the quality of voice and video in wireless networks, MOS values extending from 1 to 5 : where 1 is the exceedingly bad quality and 5 is the best quality [11]. In this simulation, we compute MOS through a non-linear mapping from Rfactor as shown below:

MOS $=1+0.035 R+7 * 10^{-6} R(R-60)(100-R)$

where

$R=100-I_{V}-I_{d}-I_{e}+A$

$\mathbf{I}_{\mathbf{v}}$ : the effect of impairments occurs with the video signal; $\mathbf{I}_{\mathbf{e}}$ : the impairments caused by different types of losses due to codec's and network, and $\mathbf{I}_{\mathbf{d}}$ : represents the impairment caused by delay particularly mouth to ear delay.

\section{F. Signal-to-Noise Ratio}

The signal to noise ratio (SNR) is the proportion between the power for the original signal and the power for unwanted signal "noise". The noise behavior and the signal to noise ratio is a basic parameter for the sight and sound media transmission services. The signal to noise ratio or SNR as it is always measured the sensitivity performance at the destination. The acceptable values for the SNR for signals are approximately above $25 \mathrm{~dB}$ for multimedia services [11].

\section{G. Bit Error Rate}

Bit error rate (BER) is characterized as the proportion between total numbers of errors to the aggregate number of transmitted bits. Bit error rate can be estimated the aggregate QoS and performance for the system including the source, destination and the control channel between the two network elements. If the channel between the source and destination is at a decent state with higher SNR, at this point the BER will be little and can be understood the best quality and services as the video and voice signals are in the best case [12].

\section{Iv. Proposed System Model}

\section{A. Simulation Parameters}

Handover process is related to access, radio resource and network control, having a significant impact on the capacity and performance of the system.

Experiment Setup: In the analysis conducted for this study, two identical scenarios were created for differentiating between $\mathrm{S} 1$ and X2 based handover over LTE-A network. one mobile user used in the two scenarios rotating with mobility feature around 4 eNodeBs at the same time, the simulation run time $=490 \mathrm{sec}$, as shown in Figure 1.

Dataset: According to Table I, II and III, In both scenarios, the two handover interfaces compared, two types of applications used Voice and Video application by three application servers connected. The network consists of four eNodeBs, parameterized. Concerning mobility, one Mobile user, and structure also includes elements EPC (Evolved Packet Core) and gateway that will communicate with the three-application server, as shown in Figure 1.

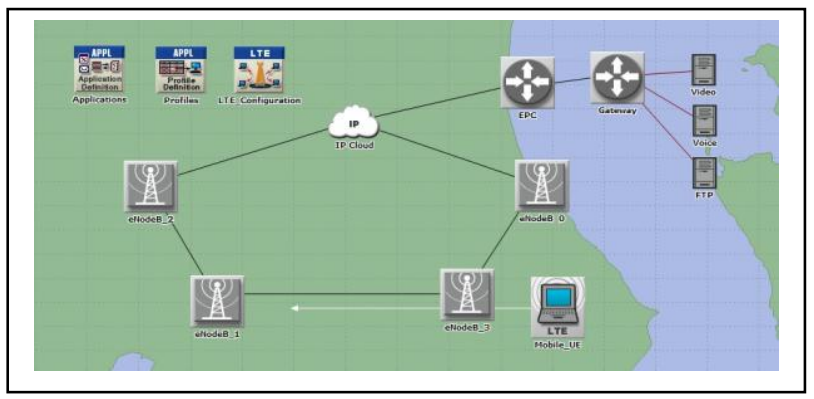

Figure 1. Network Topology for X2 and S1 Handover

\begin{tabular}{|c|l|} 
TABLE I. & VOICE PARAMETERS \\
\hline Attributes & \multicolumn{1}{|c|}{ Value } \\
\hline $\begin{array}{c}\text { Application Silence Length } \\
\text { (s) }\end{array}$ & $\begin{array}{l}\text { Exponentially distributed, mean } \\
0.65\end{array}$ \\
\hline Talk Spurt Length (s) & Exponentially distributed, mean \\
& 0.352 \\
\hline Encoder Scheme & PCM \\
\hline Voice Frames per Packet & 2 \\
\hline Type of Service & Best effort (0) \\
\hline De-Compression Delay (s) & 0.02 \\
\hline
\end{tabular}

Software Tools: In this paper, the modeling of scenarios performed using the OPNET Modeler 17.5 
Proc. of The Seventh International Conference on Advances in Computing, Electronics and Communication - ACEC 2018 Copyright (C) Institute of Research Engineers and Doctors, USA. All rights reserved.

ISBN: 978-1-63248-157-3 DOI: 10.15224/978-1-63248-157-3-11

(release 8). Performance matrices such as (End to End delay, Packet delay variation, Jitter, MOS and Traffic sent received) used in Voice and Video traffic, Signal to Noise ratio (SNR), Bit Error Rate(BER), Throughput, Downlink and Uplink Packet drop for LTE-A network.

TABLE II. LTE-A NETWORK PARAMETERS

\begin{tabular}{|c|l|}
\hline ANTENNAS Parameter & \multicolumn{2}{|c|}{ Value } \\
\hline Transmission Power & $26 \mathrm{dBm}$ \\
\hline SC-FDMA (UL) Frequency & $1710 \mathrm{MHz}$ \\
\hline $\begin{array}{c}\text { Hybrid OFDMA (DL) } \\
\text { Frequency }\end{array}$ & $2110 \mathrm{MHz}$ \\
\hline Bandwidth & $\begin{array}{l}\text { Downlink70MHz } \\
40 \mathrm{MHz}\end{array}$ \\
\hline Gain Antenna & $17 \mathrm{dBi}$ \\
\hline Antenna Height & $40 \mathrm{~m}$ \\
\hline Radius Coverage & $20 \mathrm{Km}$ \\
\hline Propagation Model & Urban \\
\hline Duration of simulation & $490 \mathrm{~s}$ \\
\hline
\end{tabular}

TABLE III. VIDEO PARAMETERS

\begin{tabular}{|c|l|}
\hline Attributes & \multicolumn{1}{c|}{ Value } \\
\hline Frame inter-arrival time information & 60 packets/sec \\
\hline Frame size information(bytes) & $128 * 240$ pixels \\
\hline Type of Service & Best effort \\
\hline
\end{tabular}

\section{B. Simulation Results and Discussions}

- Case-1: Voice

MOS: According to figure 2, it is a huge variation in the voice quality (MOS), by using $\mathrm{S} 1$, interface MOS value is about 4.4 then there is degradation up to 3.1 at the end of simulation, on the other hand, it is about 4.4 by using X2 interface all over the simulation, so it is a very good voice quality so we can conclude that X2 handover is preferred.

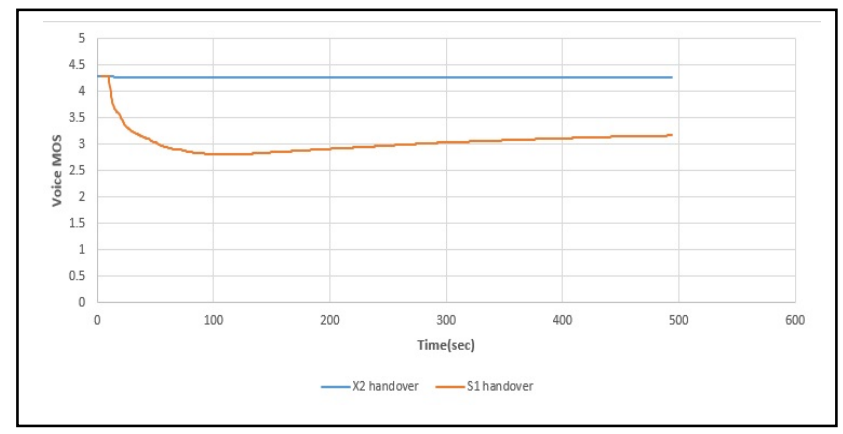

Figure 2. MOS over X2 and S1 Handover

Jitter: Figure 3 shows a very small voice jitter value approximately zero by using X2 interface compared with $0.000008 \mathrm{sec}$ using $\mathrm{S} 1$ handover, this will effect on the overall voice traffic transmitted.

End-to-End Delay (sec): As shown in figure 4, End to End delay value satisfies values near to $0.1046,0.1042 \mathrm{sec}$ in case of $\mathrm{S} 1$ and $\mathrm{X} 2$ enabled respectively at the end of simulation otherwise in figure 4, voice traffic on $\mathrm{S} 1$ interface has a higher End to End delay value than X2 interface.

Traffic sent and received (packets/sec): According to figure 5 , the average traffic sent over the two handover interfaces $\mathrm{X} 2$ and $\mathrm{S} 1$ respectively is equal to 65 (Packets/sec) at $200 \mathrm{sec}$. on the other hand figure 6 shows that the traffic received using $\mathrm{X} 2$ handover is equal about
65 (packet/sec) there are no losses using X2 handover, on the other hand by using S1 as shown there is a big packet drop in the traffic received about 10 packets dropped. Therefore, we can accumulate the packet loss ratio for each type of handover, which equal $0.05 \%$ and $6.25 \%$ for $\mathrm{X} 2$ and $\mathrm{S} 1$ handover respectively.

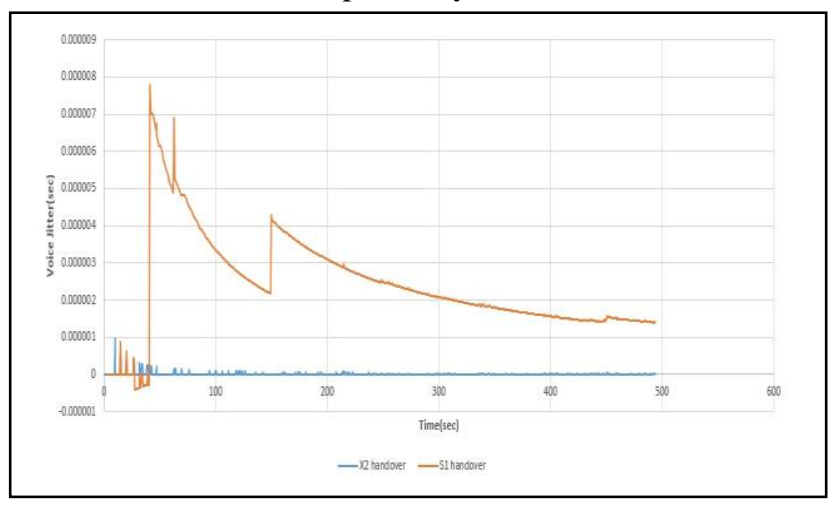

Figure 3. Jitter over X2 and S1 Handover

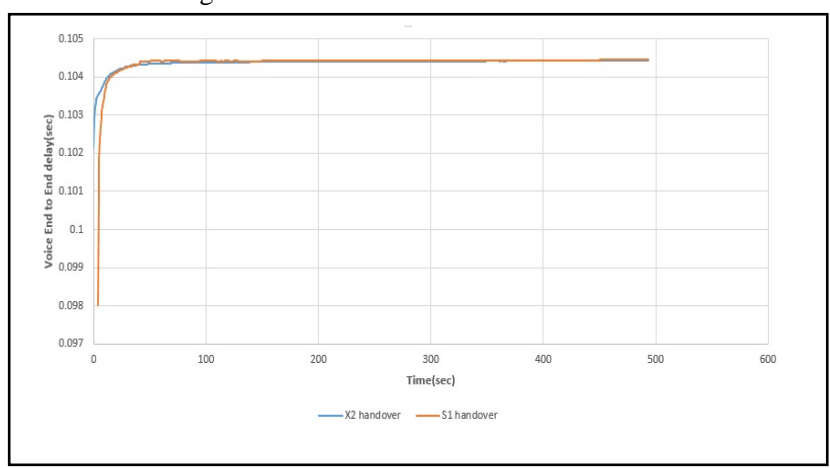

Figure 4. End-to-End Delay for X2 and S1 Handover

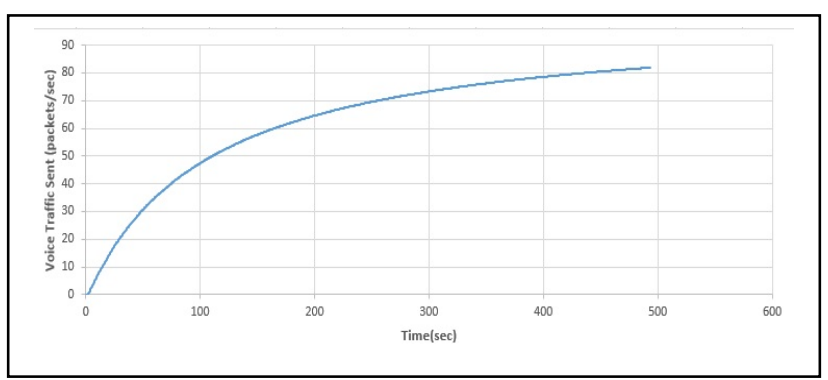

Figure 5. Traffic Sent X2 and S1 Handover (packet/sec)

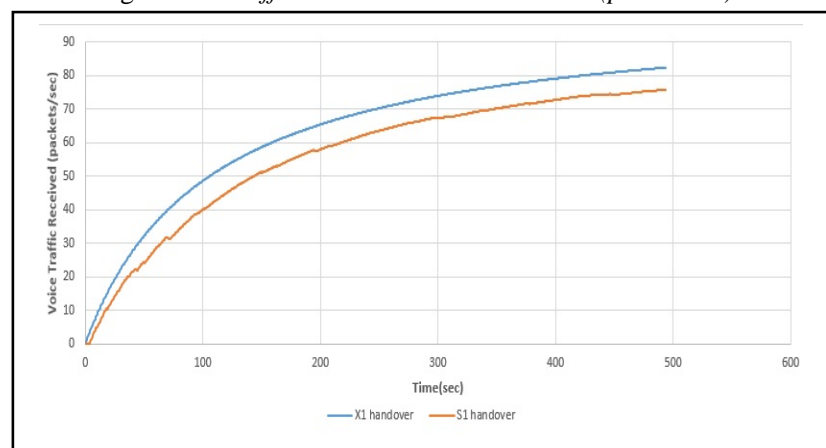

Figure 6. Traffic received X2 and S1 Handover (packet/sec)

- Case-2: Video

End to End delay (sec): Figure 7 reveals that video End-to-End delay values satisfy acceptable values not greater than $500 \mathrm{msec}$ in case of $\mathrm{S} 1$ and $\mathrm{X} 2$ enabled respectively at the end of the simulation. otherwise shown video End to End delay in S1 handover has a higher value 
Proc. of The Seventh International Conference on Advances in Computing, Electronics and Communication - ACEC 2018 Copyright (C) Institute of Research Engineers and Doctors, USA. All rights reserved.

ISBN: 978-1-63248-157-3 DOI: 10.15224/978-1-63248-157-3-11

approximately $0.044 \mathrm{sec}$ compared with X2 interface about $0.04 \mathrm{sec}$ from start to the end of simulation time.

Packet delay variation (sec): According to figure 8, The variation of packet delays satisfies very low values in case of $\mathrm{S} 1$ and $\mathrm{X} 2$ handovers, at the end of simulation time values verified $0.0000121 \mathrm{sec}$ in case of X2 handover considered as a lower value and support a fast access for the service compared with about $0.0000277 \mathrm{sec}$ for the S1 interface.

Traffic sent received (packet/sec): According to figure 9, traffic sent over LTE-A network using the two handover is equal to 10 (Packets/sec), Figure 9 shows that traffic received using $\mathrm{X} 2$ handover is equal about 10 (packet/sec) there are no packet losses, on the other hand by using $\mathrm{S} 1$ as shown there is a big packet drop in the traffic received about $40 \%$ of packets dropped at the end of the simulation. So metric of packet loss ration can be calculated at second 200 PLR will equal $32 \%$ and $0.03 \%$ by using $\mathrm{S} 1$ and $\mathrm{X} 2$ handover respectively.

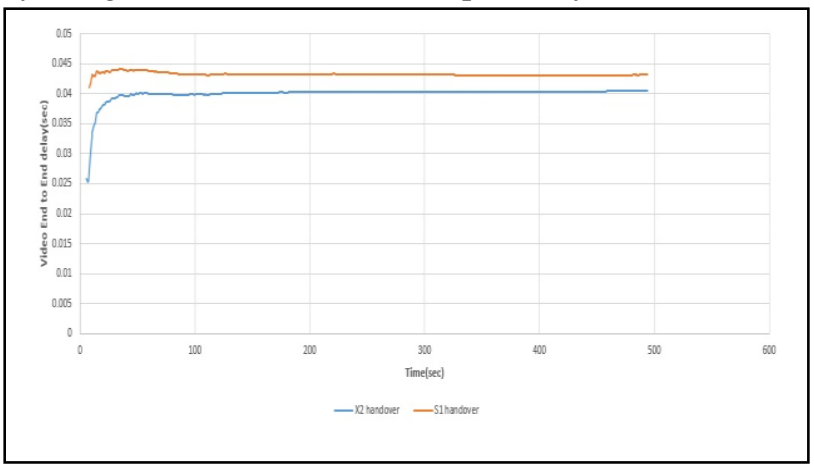

Figure 7. End-to-End Delay for X2 and S1 Handover

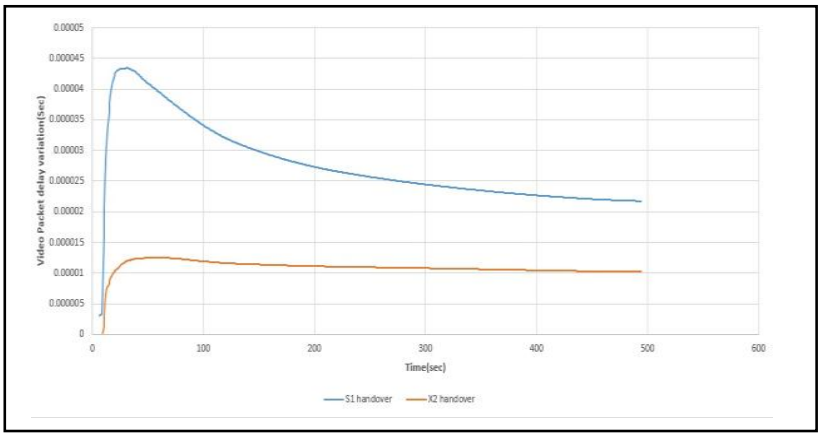

Figure 8. Packet Delay Variation for X2 and S1 Handover

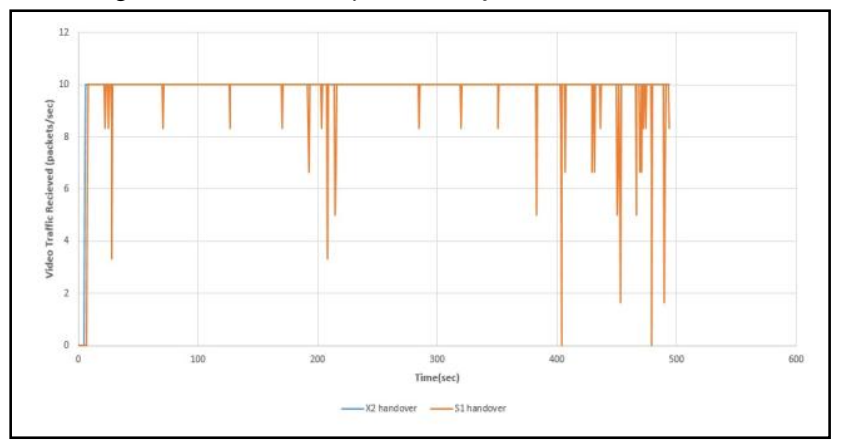

Figure 9. Traffic Received X2 and S1 Handover (packet/sec)

\section{- Case-3: HTTP}

Download Response Time and Object Response Time (sec): As shown in figure 10 and 11 respectively as shown S1 handover satisfied higher delay response values compared with the $\mathrm{X} 2$ handover values.
Traffic Sent and Received (Packet/sec): As shown in figure 12 , there is a very high data drop by using S1 handover compared with $\mathrm{X} 2$ handover known that the traffic sent is about up to 200 bytes/sec. Moreover, we can calculate the Packet Loss Ratio for the two types, at second 200 the PLR values are equal $19.7 \%$ and $0.012 \%$ for S1 and X2 handover respectively.
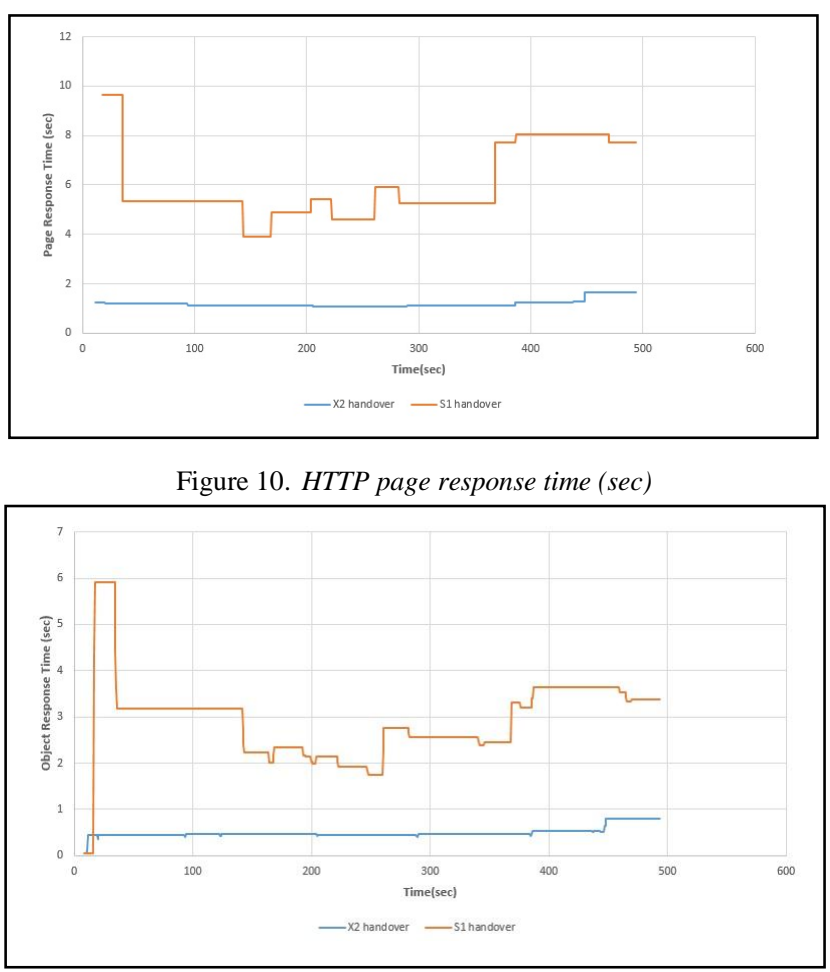

Figure 11. HTTP Object response time (sec)

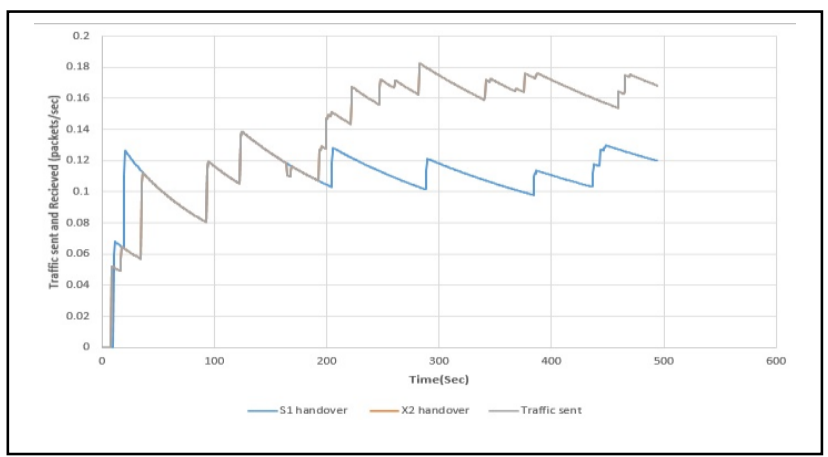

Figure 12. Traffic Sent and Received using S1 and X2 Handover

- Case-4: Network Performance

Downlink and Uplink SNR (dB): According to Figure 13, the downlink SNR is about $29 \mathrm{~dB}$ using X2 handover compared with $16 \mathrm{~dB}$ using $\mathrm{S} 1$ handover, additionally, the uplink SNR equal $25 \mathrm{~dB}$ then decreased to $12.5 \mathrm{~dB}$ at the end of simulation additionally $7 \mathrm{~dB}$ then decreased to $2.5 \mathrm{~dB}$ using X2 and S1 shown in figure 14.

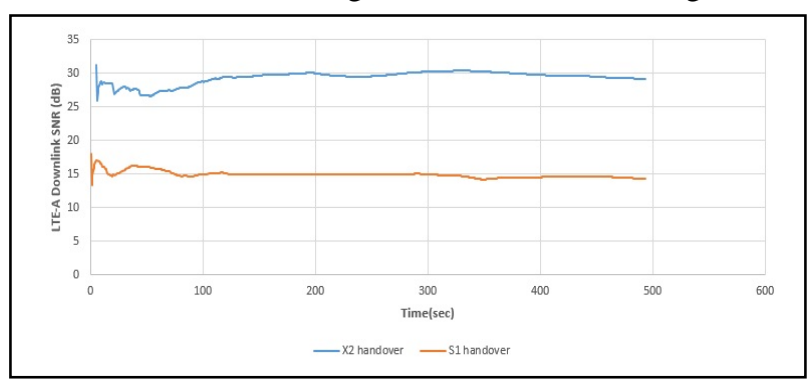

Figure 13. Downlink SNR for X2 and S1 Handover (dB) 
Proc. of The Seventh International Conference on Advances in Computing, Electronics and Communication - ACEC 2018 Copyright ( Institute of Research Engineers and Doctors, USA. All rights reserved.

ISBN: 978-1-63248-157-3 DOI: 10.15224/978-1-63248-157-3-11

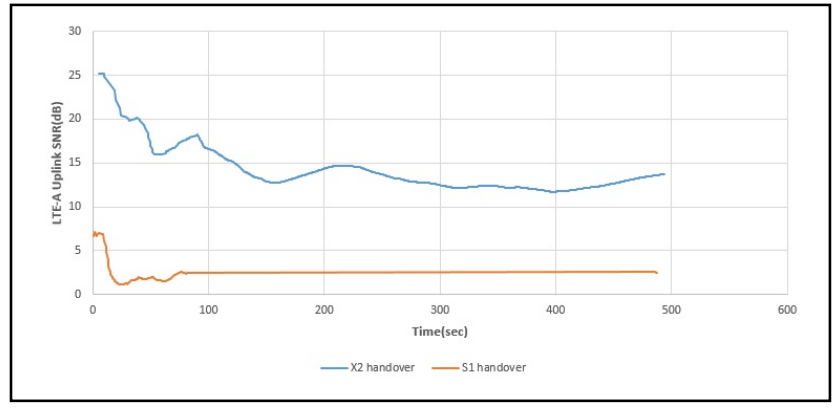

Figure 14. Uplink SNR for X2 and S1 Handover $(d B)$

BLER over Uplink and Downlink: From the simulated system according to figures 15 and 16, the accumulated Uplink and Downlink values of BER using $\mathrm{X} 2$ handover are very small approximately near to zero over Uplink and Downlink compared with S1 handover.

Throughput (Packet/sec): As shown in figure 17, by using X2 handover has a better throughput is about 65 (packets/sec) compared with S1 handover which equals 57 (packets/sec). Therefore, we can result that choosing X2 handover is a very choice compared with another one.

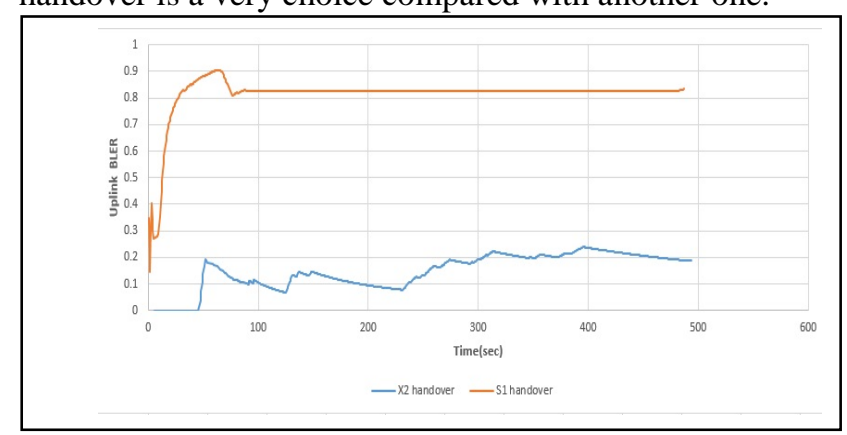

Figure 15. UL BLER for X2 and S1 Handover

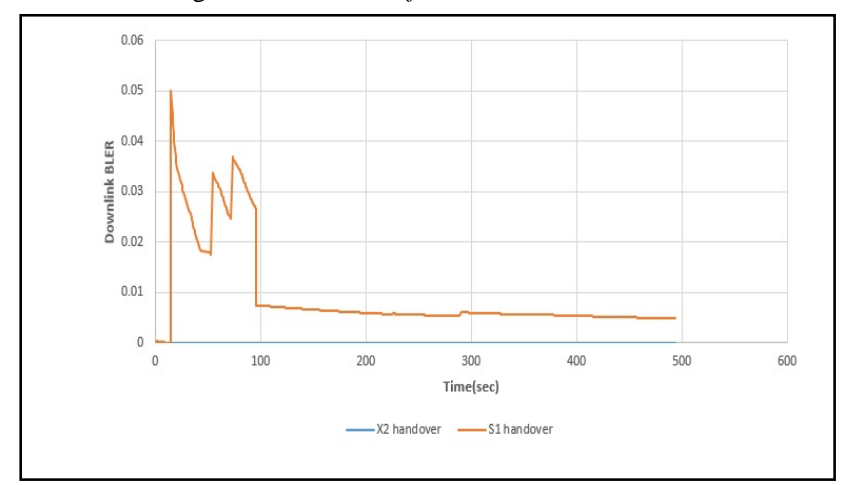

Figure 16. DL BLER for X2 and S1 Handover

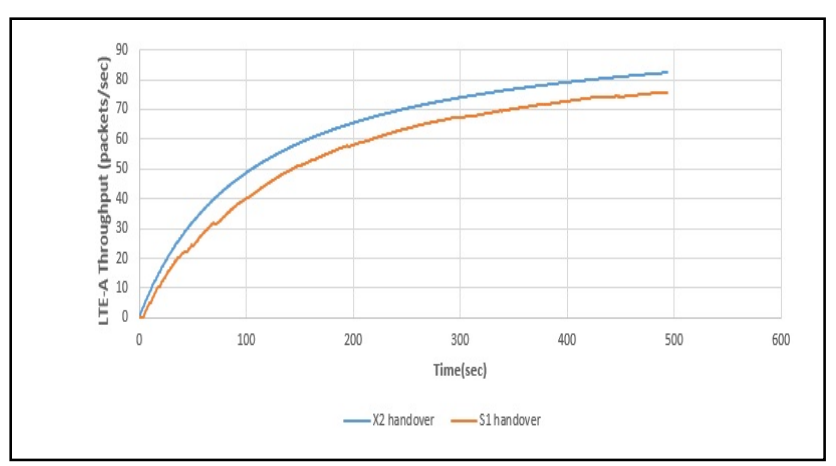

Figure 17. Throughput for X2 and S1 Handover (packets/sec)

\section{v. General Discussion}

In this case study, we focused on two main handover types, we compared between the two types in order to maintain what type is preferred on multimedia streaming and the overall performance. Also indicated that there is a better MOS, lower End to End delay, lower jitter, packet delay variation, higher traffic quality and lower packet loss ratio using X2 handover over network so there is a great improvement on the overall network performance, voice and video quality discussed in figure 18 . Additionally, this paper presented OPNET simulated networks to show the effect of using X2 handover on streaming data over LTE-A networks by realizing more real-time scenarios to verify a good empirical value based on these technology's protocols. A multimedia streaming required a high level of quality to verify the fair access for all users. More quality metrics have been investigated through the simulation of more reliable streaming data using OPNET 17.5 interface. Simulation results had shown that X2 handover increases the level of quality for overall network performance over LTE-A networks as mentioned in figure 19.

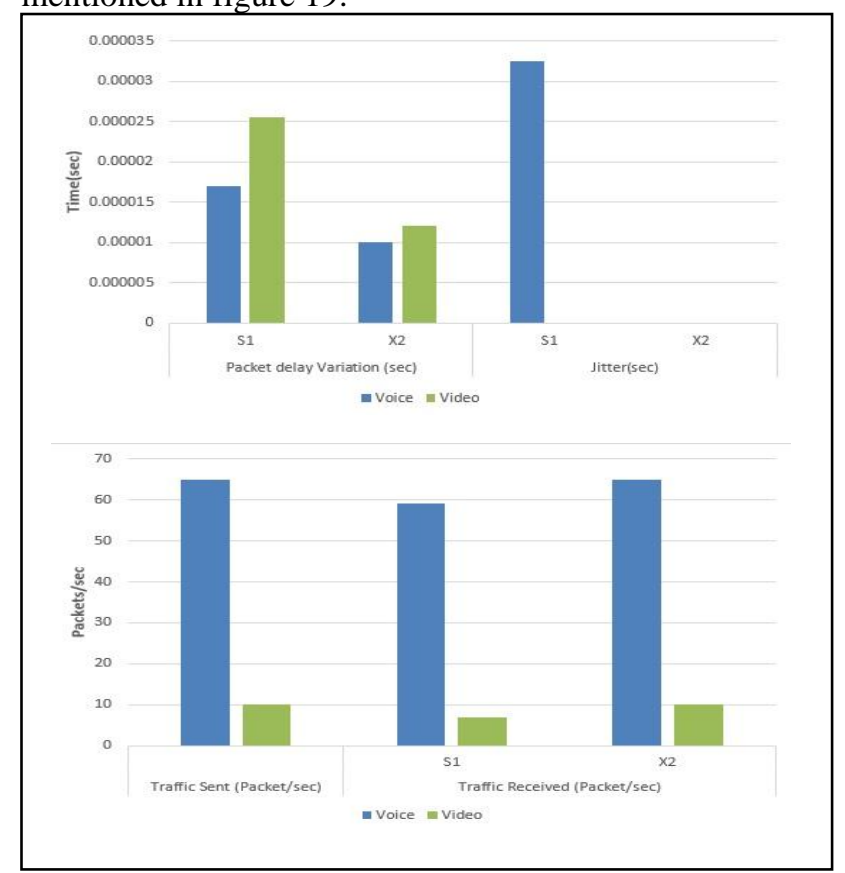

Figure 18. Video and Voice Performance Matrices Results

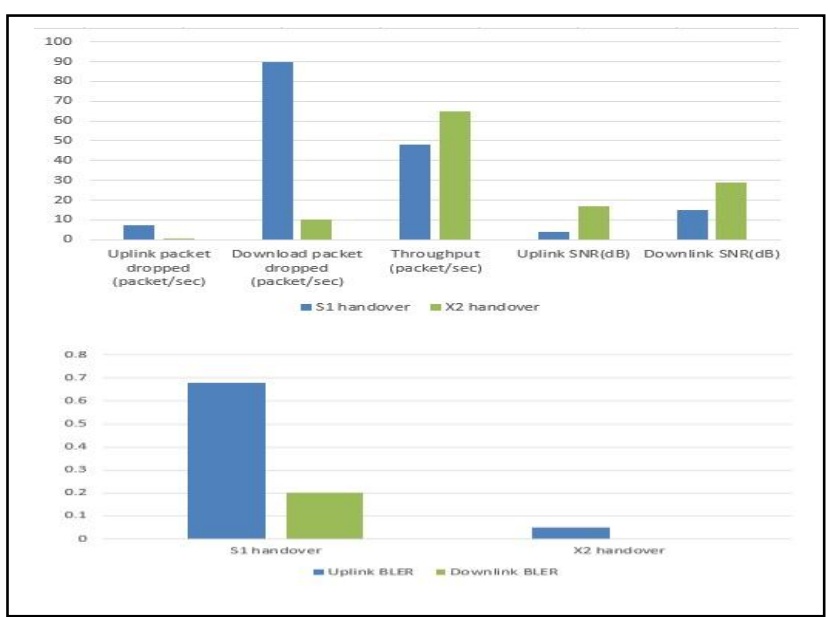

Figure 19. LTE-A network Performance Matrices Results 
Additionally, the analytical results showed that $\mathrm{X} 2$ handover besides having a lower object and page response time it also has a higher HTTP traffic received quality and lower values of packet loss ratio so it is the preferred mode discussed in figure 20.

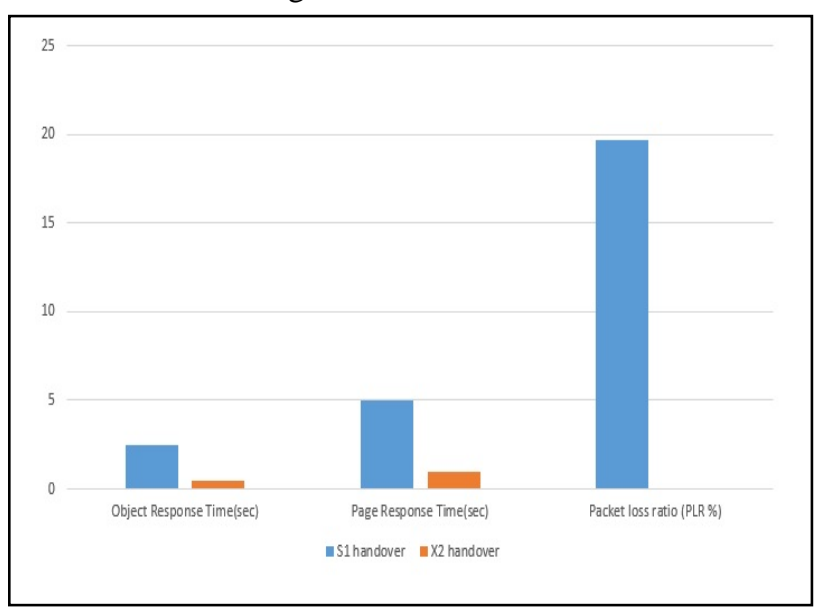

Figure 20. HTTP Performance Matrices

\section{vi. Conclusion}

Handover mechanism represents a highly effective problem in the transmission of packets for multimedia streams; so, there is the trade-off between quality and delay. The simulation results indicate that Mobile LTE-A can deliver sufficient bandwidth while ensuring that packet delays and jitter meet the requirements of multimedia streaming. This case study presents OPNET simulated networks to show the effect of handover mechanisms over LTE-A networks on multimedia streaming to satisfy a good empirical quality value on multimedia and on the overall network performance. Several important critical parameters such as end-to-end delay, packet delay variation, throughput, MOS, SNR, BER, and PLR can be analyzed. Simulation results show that by using X2 handover mechanism there is a great effect on the multimedia transmission quality and on the overall network performance compared with the S1 handover mechanism. Future work includes more suitable models for handover idea and its impact on streaming data over mobile $4.5 \mathrm{G}$ and $5 \mathrm{G}$ networks at different types of network connections under the effect of different fading effects and different paths between BSs and SSs and study different conditions for network to satisfy the best quality all the time of the service's access.

\section{References}

[1] Xiao, Qinshu, Baojiang Cui, and Lingrong Li. "An enhancement for key management in LTE/SAE X2 handover based on ciphering key parameters." P2P, Parallel, Grid, Cloud and Internet Computing, Ninth International Conference on. IEEE, 2014.

[2] Wei, Qing, et al. "Mobility management in optical mobile network." Wireless Communications and Networking Conference (WCNC), 2014 IEEE. IEEE, 2014.

[3] Pan, Meng-Shiuan, Tzu-Ming Lin, and Wen-Tsuen Chen. "An enhanced handover scheme for mobile relays in LTE-A high-speed rail networks." IEEE Transactions on Vehicular Technology 64.2 (2015): 743-756.

[4] Alexandris, Konstantinos, et al. "Analyzing x2 handover in lte/ltea." Modeling and Optimization in Mobile, Ad Hoc, and Wireless Networks (WiOpt), 14th International Symposium on. IEEE, 2016.
[5] Sun, Bangyi, et al. "A secure and effective scheme providing comprehensive forward security to LTE/SAE X2 handover key management." KSII Transactions on Internet \& Information Systems 11.9 (2017).

[6] Wang, Ying-Hong, Jui-Lin Chang, and Guo-Rui Huang. "A handover prediction mechanism based on LTE-A UE history information." Network-Based Information Systems (NBiS), 2015 18th International Conference on. IEEE, 2015.

[7] Casoni, Maurizio, et al. "Integration of satellite and LTE for disaster recovery." IEEE Communications Magazine 53.3 (2015): 47-53.

[8] Ibrahim, Ehab Ahmed, M. R. M. Rizk, and Ehab F. Badran. "Study of lte-r x2 handover based on a3 event algorithm using matlab." Information and Communication Technology Convergence (ICTC), 2015 International Conference on. IEEE, 2015.

[9] Kumar, Naresh. "Mobile Ad hoc Network: Issue and Challenges Related to QoS and Solutions." (2017).

[10] Mello, Carlos AB, et al. "A Comparative Study of Objective Video Quality Assessment Metrics." Journal of Universal Computer Science 23.5 (2017): 505-527.

[11] Sales, Martí, Md Saifuddin Faruk, and Seb J. Savory. "Improved linewidth tolerant carrier phase recovery based on polar MAP metric estimate." Optical Fiber Communications Conference and Exhibition (OFC), 2017. IEEE, 2017.

[12] Ji, Zu, Johnson O. Sebeni, and Yu-Lin Wang. "Device and method for adaptive channel estimation." U.S. Patent No. 9,819,457. 14 Nov. 2017.

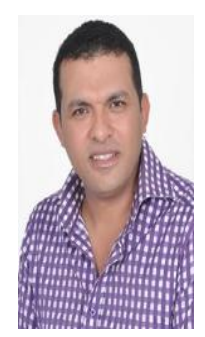

M. A. Mohamed received the Ph.D. degree in Electronics and Communications Engineering from the Faculty of Engineering - Mansoura University - Egypt by 2006. After that he worked as an assistant professor at the electronics \& communications engineering department, He had awarded the best Ph.D. thesis at Mansoura University at 2007. Then awarded the associate professor degree in 2012 until research interests are in multimedia processing, wireless communication systems, and field programmable gate array (FPGA) applications. Now he is a professor and chairman at the Electronics and Communications Department in Mansoura University since 2017. He has 150 publications in various international journals and conferences. His current res research interests are in multimedia processing, wireless communication systems, and field programmable gate array (FPGA) applications.

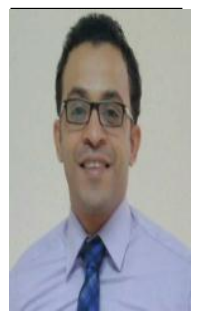

H.A. Sakr received the M.Sc. in Communications and Electronics Engineering from the Faculty of Engineering - Mansoura University - Egypt by 2014 and the B.Sc. degree in Electronics and Communications Engineering from 10th of Ramadan Engineering faculty in 2009. He worked as an assistant lecturer at Institute of Public Administration - Electronics and Communications department - Saudi Arabia. 